\title{
Anthocyanins and intestinal barrier function: a review
}

\begin{abstract}
Jonathan C. Valdez and Bradley W. Bolling*
Department of Food Science, University of Wisconsin-Madison, 1605 Linden Dr., Madison, WI 53706, USA

*Corresponding author: Bradley W Bolling, 1605 Linden Dr. Madison, WI 53706, USA. Tel: 608-890-0212; E-mail: bwbolling@wisc.edu DOI: $10.31665 / J F B .2019 .5175$

Received: December 15, 2018; Revised received \& accepted: December 31, 2018

Citation: Valdez, J.C, and Bolling, B.W (2019). Anthocyanins and intestinal barrier function: a review. J. Food Bioact. 5: 18-30.

Abstract

Chronic intestinal inflammation, occurring in inflammatory bowel diseases (IBD), is associated with compromised intestinal barrier function. Inflammatory cytokines disrupt tight junctions and increase paracellular permeability of luminal antigens. Thus, chronic intestinal barrier dysfunction hinders the resolution of inflammation. Dietary approaches may help mitigate intestinal barrier dysfunction and chronic inflammation. A growing body of work in rodent models of colitis has demonstrated that berry consumption inhibits chronic intestinal inflammation. Berries are a rich dietary source of polyphenolic compounds, particularly anthocyanins. However, berry anthocyanins have limited bioavailability and are extensively metabolized by the gut microbiota and host tissue. This review summarizes the literature regarding the beneficial functions of anthocyanin-rich berries in treating and preventing IBD. Here, we will establish the role of barrier function in the pathogenesis of IBD and how dietary anthocyanins and their known microbial catabolites modulate intestinal barrier function.
\end{abstract}

Keywords: Colitis; Barrier function; Anthocyanin; Metabolism; Inflammatory Bowel Diseases.

\section{Introduction}

Inflammatory Bowel Diseases (IBD) refer to a set of multifactorial disorders of the gastrointestinal tract differentiated as ulcerative colitis (UC) and Crohn's disease (CD). IBD are characterized by an abhorrent inflammatory response resulting in alternating phases of clinical relapse and remission (Ahluwalia et al., 2018). Dietary factors have been implicated in the pathogenesis and prevention of IBD, but effective dietary recommendations for patients with IBD are not yet available (Cohen et al., 2013).

Anthocyanin-rich berries have emerged as a promising dietary intervention in animal models of IBD and pilot human studies (Biedermann et al., 2013; Martin and Bolling, 2015). Describing the specific mechanisms by which berry bioactives may inhibit intestinal inflammation is necessary to develop effective dietary recommendations for IBD.

Intestinal barrier dysfunction is common in IBD and a central mechanism by which dietary bioactives may mitigate colitis. Polyphenols are promising bioactives to improve intestinal barrier function and prevent colitis (Murphy et al., 2018; Wu et al., 2018). However, berry anthocyanins are extensively metabolized by host tissue and gut microbiota. These catabolites also have variable absorption and intrinsic anti-inflammatory activity (Wang et al., 2018; Wu et al., 2018). Therefore, the objectives of this review are to describe the essential components of intestinal barrier function in the context of chronic inflammation and to elaborate the means by which berry anthocyanins and its known catabolites could inhibit IBD by improving intestinal barrier function.

\section{Tight junction proteins modulate intestinal permeability}

The outermost layer of cells in the gastrointestinal tract is organized in a single layer dominated by columnar absorptive enterocytes. While these cells are relatively impermeable to most luminal antigens, their intercellular spaces provide a weak point to barrier function. To minimize paracellular flux, intestinal epithelial cells form intercellular junctional complexes. These molecular gates can be viewed as four separate parts: the tight junction (TJ), adherens junction, gap junction and the desmosome. Together with the adherens junction, TJ complexes bind epithelial cells together via the actin cytoskeletons of adjoining cells (Ulluwishewa et al., 


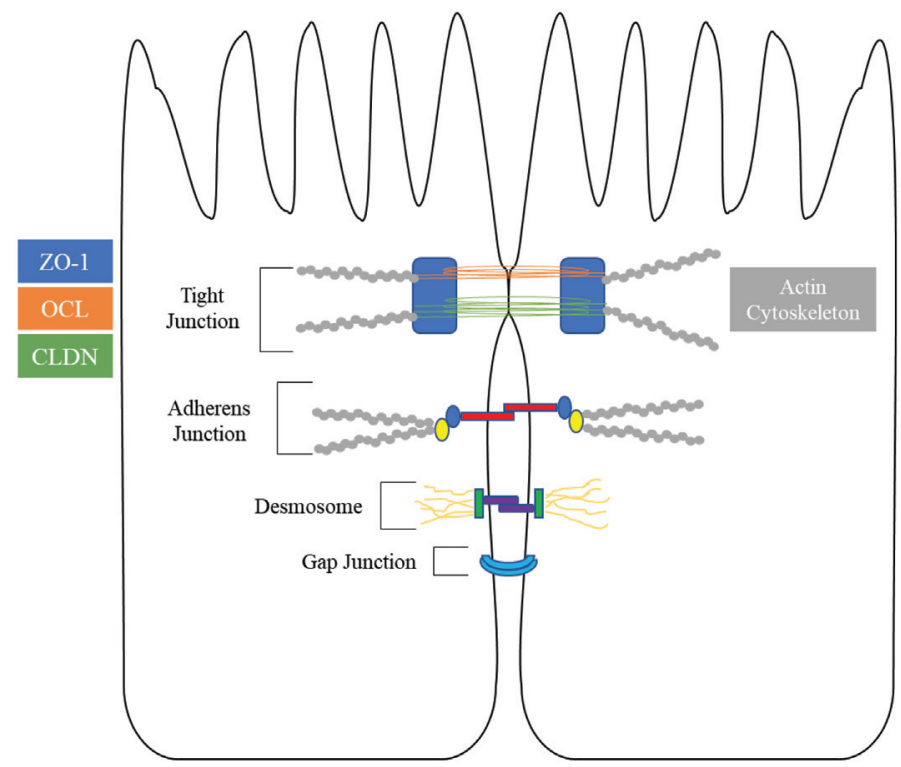

Figure 1. Overview of tight junction proteins in enterocytes (Chiba et al., 2008; Michielan and D'Incà, 2015; Ulluwishewa et al., 2011).

2011).

Maintaining the integrity of the intestinal epithelial barrier is essential for immune homeostasis and the prevention of chronic intestinal inflammation. Early tight junction modeling assumed a simple static paracellular barrier. However, it is now understood that epithelial barrier function is dynamic and regulated by $\mathrm{TJ}$ acting in concert with the immune system (Liang and Weber, 2014).

$\mathrm{TJ}$ control of paracellular flux across the epithelium can be categorized as contributors to "pore" and "leak" pathways. TJ pores act as charge and size-selective conduits and are generally formed by claudins. The abundance and localization of these pore-forming claudins, allow epithelial cells to control the flux of small molecules through the paracellular spaces. Leak pathways have not been characterized to the same extent but involve physical changes to the overall tight junction architecture. Strand breaks and transient paracellular gaps result in an increased, non-selective flux of larger molecules (Garcia-Hernandez et al., 2017).

\subsection{Zonula occludens}

Zonula occludens (ZO) serve as an intermediary between transmembrane TJ proteins and the actin cytoskeleton (Lee, 2015). Specifically, ZO interfaces with the perijunctional acto-myosin ring, a belt-like structure that encircles the apical pole of epithelial cells (Figure 1). Through the contraction of this ring epithelial cells can control the maintenance and regulation of tight junctions (Furuse, 2010; Lee, 2015). Plaque proteins are characterized by the presence of PSD95-DlgA-ZO-1 homology (PDZ) domains, which can associate with other PDZ domains or specific C-terminal sequences of transmembrane proteins (Fanning et al., 1999).

Amongst this class of proteins, the function and structure of ZO-1 has been extensively characterized. ZO-1 serves as a central hub which may facilitate connection of the various parts of tight junction complexes (Lee, 2015). Through multiple PDZ domains, ZO-1 can interact with claudin, occludin, and JAM, while simultaneously forming dimers with ZO-2 and ZO-3 (Raleigh et al., 2011).

\subsection{Occludin}

Occludin is a $60 \mathrm{kDa}$ transmembrane protein and its function in modulating intestinal permeability is still not fully understood (Chiba et al., 2008). The $\mathrm{NH}_{2}$-terminus and extracellular loop of occludin appears to be essential in maintaining and regulating barrier function (Bamforth et al., 1999). Occludin may not be essential for intestinal barrier formation and maintenance, but rather may regulate the pore and leak pathways via interaction with claudins (Van Itallie et al., 2010).

\subsection{Claudins}

Claudins are a class of 26 transmembrane proteins, ranging from 18 to $27 \mathrm{kDa}$ that form the backbone of TJs (Garcia-Hernandez et al., 2017). The C-terminal region of claudins bind PDZ domains that facilitate interaction with plaque proteins, a requirement for localization and stabilization at epithelial TJs (Ruffer and Gerke, 2004). In addition to their integral role in barrier function, claudins are also able to facilitate the selective flux of molecules through the paracellular space (Lingaraju et al., 2015). Claudins are categorized into two groups dependent on their function. The "tight" claudins, including claudins-1, 3, 4, 5, 6, 12, 18, and 19, serve to seal TJs (Garcia-Hernandez et al., 2017). The "leaky" claudins form pores in the intercellular space, allowing for increased paracellular permeability to water and small ionic solutes. This group includes claudins-2,7, 8, 13 and 14 (Fujita et al., 2006).

These categorizations, while useful, are an oversimplification. The function of a particular claudin is determined by its interaction with other claudins in the TJ. For example, claudin- 4 has been classified as functioning as both a leaky and tight TJ protein (Garcia-Hernandez et al., 2017).

\section{Chronic inflammation drives barrier dysfunction in IBD}

The intestinal epithelium is the physical barrier that separates mi- 
crobes and antigens in the lumen from the mucosal immune system. Disruption of barrier function is a common feature of IBD, but whether it is a causal factor, or merely a symptom of chronic inflammation is unclear (Michielan and D'Incà, 2015). Impaired barrier function has been reported in tissue of patients with minimal mucosal damage, suggesting that epithelial barrier dysfunction contributes to the initiation of inflammation in certain subsets of IBD (Gersemann et al., 2008).

Inflammation associated with IBD disrupts the epithelial barrier resulting in a heightened immune response (Ahluwalia et al., 2018). Increased levels of pro-inflammatory components stimulate further loss of the mucosal barrier (Abraham et al., 2017). Normal immunoregulatory function, which could moderate the severity and longevity of the immune response is also impaired in IBD (Michielan and D'Incà, 2015). Although the inflammatory response is complicated, there are common pathways and causal factors linking IBD, inflammation, and increased intestinal permeability.

\subsection{Mechanisms of inflammatory-mediated barrier dysfunc- tion}

\subsubsection{NF-KB pathway}

Nuclear-factor kappa-light-chain-enhancer of activated B cells $(\mathrm{NF}-\mathrm{\kappa B})$ is a central pathway involved in maintaining immune homeostasis. Inappropriate activation and expression of NF-kB p65 protein leads to chronic inflammation and is positively correlated with the severity of inflammation and neutrophil inflammation in

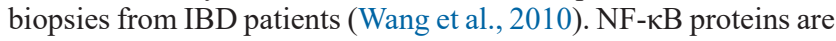
found in the cytosol bound to NF- $\kappa B$ inhibitor I- $\kappa B$. Degradation or depletion of $\mathrm{I}-\kappa \mathrm{B}$, results in translocation of NF- $\kappa \mathrm{B}$ to the nucleus, resulting in elevation of pro-inflammatory cellular response. Initiation of NF- $\kappa \mathrm{B}$ can induce the production pro-inflammatory mediators and influence differentiation of innate and adaptive immune cells towards an inflammatory state (Sen and Baltimore, 1986). NF- $\mathrm{B}$ B activation can increase paracellular permeability by promoting the internalization of tight junction proteins and upregulating pore-forming claudin-2 in intestinal epithelial cells (Kucharzik et al., 1998; Tang et al., 2010).

\subsubsection{Myosin light-chain kinase}

Myosin light-chain kinase (MLCK) serves as the conduit for cellular control epithelial barrier function via the actomyosin ring (U1luwishewa et al., 2011). In active IBD, overexpression contributes to a sustained reduction in barrier function, and therefore chronic inflammation (Zolotarevsky et al., 2002). MLCK interacts with the equatorial actomyosin ring by catalyzing the phosphorylation of myosin II regulatory light chain (Blair et al., 2006). Subsequently, the organization of occludin and $\mathrm{ZO}-1$ at the $\mathrm{TJ}$ is altered, leading to increased paracellular permeability (Shen et al., 2006).

\subsubsection{Mitogen-activated protein kinases}

The mitogen-activated protein kinase (MAPK) signal transduction pathways are driven by serine/threonine specific protein kinases which are associated with the initiation of chronic inflammation in IBD (Broom et al., 2009). Elevated expression of extracellular-regulated kinase (ERK) 1/2 has been reported in IBD and its activation increases significantly in the active state of the disease
(Broom et al., 2009). ERK 1/2 can decrease epithelial permeability via interaction with $\mathrm{TJ}$ proteins and the actin cytoskeleton and inhibit oxidative stress-induced barrier dysfunction in vitro (Basuroy et al., 2006) Additionally, MAPK p38 $\alpha$ expression is reported to coincide with presence and severity of inflammation in active IBD (Waetzig et al., 2002).

3.1.4. Cytokines affect barrier function through regulation of cellular immunity

Cytokines are a class of regulatory proteins that define the immune response and impact epithelial barrier function (Turner et al., 2014). The cytokine profiles of IBD are characterized by an imbalance favoring pro-inflammatory cytokines over their immunomodulatory counterparts. The proinflammatory cytokines tumor necrosis factor- $\alpha$ (TNF- $\alpha)$, interferon- $\gamma$ (IFN- $\gamma)$, Interleukin- $1 \beta$ (IL-1 ), IL-17A, IL-6, IL-12, IL-18, IL-21, IL-23, IL-27, and IL32 are associated with inflamed tissue and common in both forms of IBD (Nemeth et al., 2017; Velikova et al., 2017). The cytokine profiles of CD and UC differ by the magnitude of Th1/Th2/Th17 inflammatory responses. While CD is associated with a Th1/Th17 pro-inflammatory response, $\mathrm{UC}$ is characterized by increased $\mathrm{Th} 2$ associated cytokines (Nemeth et al., 2017).

Immunoregulatory cytokines, such as IL-10 and IL-22 are critical in maintaining immune homeostasis and their dysregulation is associated with IBD pathogenesis. Reduction or dysfunction of these immunomodulators contribute to abhorrent immune response and therefore epithelial barrier function (Marlow et al., 2013). The interplay between barrier function and cytokines is complex and highly dependent on the broader context of inflammation. A full-review of our current knowledge of cytokine-induced inflammation and barrier dysfunction is beyond the scope of this review. We will instead focus on three well-characterized cytokines, TNF- $\alpha$, IL- $1 \beta$, and IFN- $\gamma$.

\section{TNF- $\alpha$}

TNF- $\alpha$ is strongly associated with both UC and CD (Ahluwalia et al., 2018). This cytokine has a marked effect on barrier function, exerting its effects via activation of NF- $\mathrm{KB}$ and MLCK (Graham et al., 2006; Ma et al., 2004). In a monolayer of colonic epithelial cells, TNF- $\alpha$ exposure for 24 hours reduced the transepithelial electrical resistance (TEER) by $80 \%$ (Schmitz et al., 1999). In vitro experimentation has implicated the observed reduction of ZO-1 and occludin, disassembly of TJ architecture, upregulation of pore-forming claudins, and rearrangement of the actin cytoskeleton (Schmitz et al., 1999) (Figure 2).

\section{IFN- $\gamma$}

IFN- $\gamma$ is a pro-inflammatory cytokine mostly associated with $\mathrm{CD}$ (Stallmach et al., 2004). Excessive IFN- $\gamma$ exposure results in the reorganization and modified expression of TJ proteins (ZO-1, ZO2 , and occludin) as well as modification of the actomyosin cytoskeleton (Bruewer et al., 2005). Furthermore, IFN- $\gamma$ enhances the barrier disrupting properties of TNF- $\alpha$ in vitro, doubling the reduction in TEER induced with TNF- $\alpha$ alone (Fish et al., 1999).

\section{$\mathrm{IL}-1 \beta$}

IL- $1 \alpha$ and IL- $1 \beta$ are mediators of the acute phase of IBD (Vounot- 


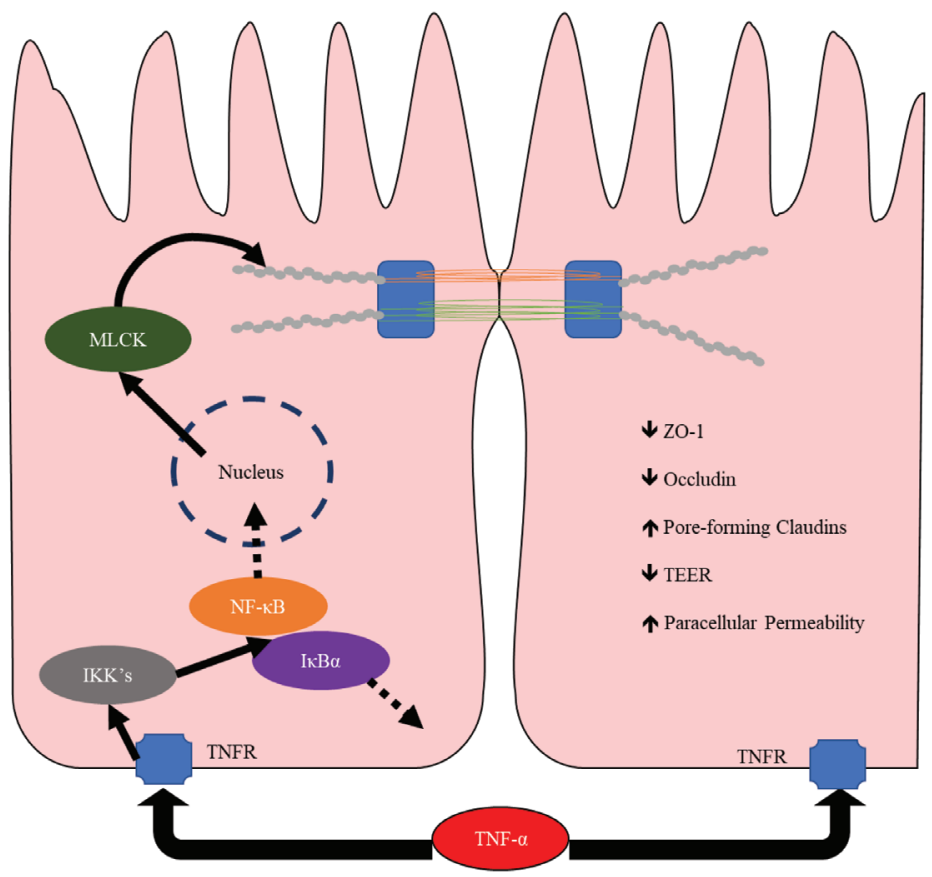

Figure 2. Mechanism by which TNF- $\alpha$ modulates tight junctions in epithelial cells. Abbreviations: MLCK, myosin light-chain kinase; IKK, IKB kinase; IKB $\alpha$, inhibitor of kappa B; TNFR: tumor necrosis factor receptor; TNF- $\alpha$, tumor necrosis factor- $\alpha$; ZO-1, zonula occludens-1; TEER, trans-epithelial electrical resistance. Adapted from Ulluwishewa et al. (2011) and Weber et al. (2010).

rypidis et al., 2013). In the context of IBD, IL-1 $\alpha$ seems to be associated with $\mathrm{CD}$, while IL-1 $\beta$ expression is enhanced in active UC (Vounotrypidis et al., 2013). IL-1 $\beta$ induces the delocalization of ZO-1 and occludin from the TJ, eliminating their effectiveness in maintaining the intestinal barrier. This is accomplished via activation of MAPK ERK kinase kinase 1 (MEKK1), which in turn activates NF- $\kappa$ B and MLCK (Kimura et al., 2009).

\section{Impact of gut dysbiosis on chronic inflammation}

In addition to the genetic factors of the host, the gut microbiome plays an integral role in chronic inflammation and barrier dysfunction (Lane et al., 2017). The healthy gut maintains homeostasis between the microbiota and mucosal immunity. Pathological alterations to the gut microbial ecology, or dysbiosis, trigger an excessive immune response in some individuals. In addition to the subsequent disruption of intestinal barrier function, a heightened immune response further alters the gut microbial populations (Lane et al., 2017). These alterations may increase the relative pathogenicity of the microbiome, contributing to further disease progression (Shen et al., 2018).

Alterations in mucosal associated microbiota are apparent in a subset of IBD cases (Morgan et al., 2012; Sepehri et al., 2007). Several studies correlate a reduction in richness and evenness of mucosal-associated microbial population with the active state of disease (Manichanh et al., 2006; Ott et al., 2004; Sepehri et al., 2007). Loss of species diversity, as well as loss of functional diversity within the microbiome correspond with IBD severity (Le Chatelier et al., 2013; Morgan et al., 2012). IBD is also associated with the decreased relative abundance and diversity within phylum Firmicutes and may contribute to the initiation and/or progression of disease (Manichanh et al., 2006).

\section{Berry anthocyanins}

Many berries are rich sources of anthocyanins. While the anthocyanin profile differs between berries, glycosylated cyanidins are the prevalent anthocyanin reported in the literature (Rothwell et al., 2013). Less prevalent berry anthocyanins includedelphinidin, malvidin, pelargonidin, peonidin, and petunidin.

The most commonly consumed berries in the U.S. are blueberry, strawberry, and red raspberry, which have 150-164, 73 , and $43 \mathrm{mg}$ anthocyanins per $100 \mathrm{~g}$ f.w., respectively (Rothwell et al., 2013). Other underutilized berries such as aronia berry, elderberry, black currant, and black raspberry have much higher anthocyanin content, $>820 \mathrm{mg}$ anthocyanin per $100 \mathrm{~g}$ f.w. (Rothwell et al., 2013).

\section{Berries and anthocyanins for IBD}

A growing number of studies have characterized the effects of anthocyanin-rich berries and their polyphenol isolates on IBD. Bilberries, raspberries, blueberries, blackberries, black currants, wine extract, pomegranate, cranberries and grapes have exhibited therapeutic potential for IBD (Deiana et al., 2012; Jung et al., 2015; Kim et al., 2017; Romier-Crouzet et al., 2009; Roth et al., 2016; Xiao et al., 2015; Yu et al., 2011). Research approaches utilizing cell culture, animal models, and human participants have helped to elucidate the mechanisms by which anthocyanin-rich fruits may prevent and ameliorate chronic intestinal inflammation.

\subsection{Modulation of oxidative stress}

Polyphenols may inhibit inflammation in the gut through direct or indirect mechanisms that reduce oxidative stress. Reactive oxygen 
species (ROS) and reactive nitrogen species (RNS) are produced in excess during chronic inflammation, disrupting redox homeostasis (Tian et al., 2017). In IBD, intestinal epithelial cells along with neutrophils and macrophages generate superoxide and nitrous oxide, potent ROS/RNS, in response to pro-inflammatory cytokines. The dysregulation of homeostasis results in increased paracellular permeability as well as direct cellular damage due to protein, lipid, and nucleic acid oxidation. (Banan et al., 1999; Rao et al., 1999).

Early research described the ability of berries to inhibit the redox dysregulation, therefore preventing the initiation of this disruptive cascade. For example, Caco-2 cells exposed to tert-butyl hydroperoxide $(\mathrm{TBH})$ simulates oxidative stress, resulting in a significant decrease in TEER. Wine extracts inhibited the effects of TBH on the cells, which led the authors to posit the direct antioxidant potential of polyphenol-rich supplements (Deiana et al., 2012). However, direct antioxidant activity is expected to have a limited role in barrier function due to the low bioavailability, and stability, as well as extensive biotransformation of anthocyanins (Hollman et al., 2011).

\subsection{Modulation of cellular antioxidant function}

Berries and their constituent polyphenols can improve antioxidant function of tissues via cellular mechanisms. For example, Rubus fruits (blackberries, and black and Korean raspberries) improved the antioxidant function of cultured intestinal cells (Jung et al., 2015). Cyanidin-3-glucoside (Cy3Glu)-rich rubus extracts reduced inducible nitric oxide synthetase (iNOS) and cyclooxygenase-2 (COX-2), enzymes responsible for ROS/RNS production in inflamed Caco-2 cells (Jung et al., 2015). Similarly, extracts of blueberry and pomegranate were able to reduce the expression of iNOS and COX-2 in mouse models of colitis (Kim et al., 2017; Pervin et al., 2016). Administration of blueberry extract also reduced neutrophil infiltration in colonic tissue, further preventing redox perturbation (Pervin et al., 2016).

\subsection{Nuclear factor erythroid 2-related Factor (Nrf2)}

Polyphenols may improve cellular antioxidant function via nuclear factor erythroid 2-related factor (Nrf2) (Jung et al., 2015). Nrf2 is a transcription factor which activates cellular antioxidant systems and dysregulation of this protein is associated with increased inflammatory response (Gerstgrasser et al., 2017) Polyphenols, including quercetin, rutin, and caffeic and ferulic acids, reversed depression of Nrf2 activation by Kelch-like ECH-associated protein 1 (KEAP1) in hepatic cells (Bayele et al., 2016). After traumatic brain injury, Nrf2-deficient mice experienced higher levels of NF- $\mathrm{B}$ activation resulting in increased intestinal permeability (Jin et al., 2008).

Purified anthocyanins also modulate cellular antioxidant function by activating Nrf2. Cy3Glu pretreatment reduced inflammation in Caco-2 cells exposed to TNF- $\alpha$ (Ferrari et al., 2016). $\mathrm{NF}-\kappa \mathrm{B}$ inhibition and modulation of the of Nrf2 were identified as possible mechanisms (Ferrari et al., 2016). Furthermore, Cy3Glu exposure increased Nrf2 activation in HT-29 cells stimulated with pro-inflammatory cytokines IL- $1 \alpha$, TNF- $\alpha$, and IFN- $\gamma$ (Serra et al., 2016). The upregulation of Nrf2 decreased NF-kB activation and inhibited cytokine induction of iNOS, COX-2 and IL-8 in HT-29 cells (Serra et al., 2016).

\section{4. $N F-K B$}

Depletion of Nrf2, antigen receptor activation and interaction with proinflammatory cytokines are factors which can initiate NF- $\mathrm{BB}$ activation (Jung et al., 2015; Serra et al., 2016). Activation of Nrf2 in LPS-stimulated Caco-2 cells treated with polyphenol rich extracts of rubus fruits, also decreased activated NF- $\mathrm{kB}$. Additionally, anthocyanins inhibited LPS-induced degradation of I- $\kappa$ B $\alpha$ (Jung et al., 2015). NF- $\mathrm{BB}$ inhibitory potential of anthocyanin-rich berry extracts has also been observed in human epithelial cell models stimulated by proinflammatory cytokines (TNF- $\alpha$, IL- $1 \beta$, and IFN- $\gamma$ ) (Kuntz et al., 2015; Nunes et al., 2013; Taverniti et al., 2014).

\subsection{Cellular kinases}

Enhancement or protection of intestinal epithelial barrier function by anthocyanins is dependent on the activities of cellular kinases. Anthocyanins isolated from Meoru, a Korean fruit, increased TEER in HCT-116 human colon cancer cells via the activation of p38-MAPK, a kinase which contributes to the sealing of epithelial TJ (Shin et al., 2011). p38-MAPK activity is increased in patients with IBD, and intestinal epithelial cells are susceptible to its direct or indirect effects (Feng and Li, 2011). Pomegranate extract reduced the phosphorylation of ERK $1 / 2$, another member of the MAP kinase family, in IL- $1 \beta$ stimulated cells (Romier-Crouzet et al., 2009). In a DSS-induced murine model of colitis, administration of an anthocyanin-rich extract of red raspberries reduced histological damage, as well as colitic wasting (Li et al., 2014). The protective effects of anthocyanins were attributed to the modulation of c-Jun N-terminal kinase (JNK) (Li et al., 2014).

\subsection{Modulation of cytokines}

Through interaction with various inflammatory pathways, anthocyanins ultimately modulate cytokine expression and signaling. Anthocyanin-rich extracts from pomegranate and red wine reduced expression of pro-inflammatory IL-8 in cytokine stimulated epithelial cells in vitro (Nunes et al., 2013; Romier et al., 2008). Pomegranate extract reduced levels of TNF- $\alpha$, IL-1 $\beta$, and IL- 6 in intestinal tissue in a DSS-colitis mouse model (Kim et al., 2017). Blueberry extract reduced TNF- $\alpha$, IFN- $\gamma$, and IL-12 and normalized IL-10 expression in the colonic mucosa of mice treated with 2,4,6-trinitrobenzenesulfonic acid (TNBS) (Yu et al., 2011). In UC patients, consumption of an anthocyanin-rich bilberry extract reduced pro-inflammatory TNF- $\alpha$ and IFN- $\gamma$ and increased immunoregulatory IL-10 in colonic tissue (Roth et al., 2016). IL-17A and IL-22, potential immunoregulatory cytokines, production was also upregulated in response to bilberry anthocyanins (Roth et al. 2016). Anthocyanins may also antagonize cytokine receptors directly and modulate the downstream effects of cytokine signaling. For example, cyanidin and its glycosides bind directly to the IL17 receptor and inhibit IL-17A signaling (Liu et al., 2017). These results suggest that dietary anthocyanins may affect the intestinal barrier in IBD by regulating the effects of cytokines on inflammatory pathways.

\subsection{Inhibition gut microbiota dysbiosis}

A majority of dietary phenolics reach the colonic microbiota intact, where they are extensively catabolized by the resident microflora. Reciprocally, dietary compounds can have a profound effect on the makeup of these microbial communities. Polyphenol-rich foods can modulate gut microbial populations ameliorating or prevent- 
ing dysbiosis in IBD (Willson and Situ, 2017). Although a comprehensive characterization of the gut microbiome in health and disease has yet to be established, there are some trends that may help in associating dietary changes to possible therapeutic benefits (Mosele et al., 2015). Decreased diversity, reduction in functionality, and an imbalance in harmful and beneficial bacterial strains are all associated with dysbiosis of the gut microbiota (Le Chatelier et al., 2013; Mosele et al., 2015).

Polyphenols, and particularly anthocyanins, may be therapeutic to the dysbiotic gut by selectively inhibiting or enhancing the growth of commensals. Glucosides of malvidin, cyanidin, delphinidin, and petunidin can enhance the relative abundance of bifidobacteria, enterococci and lactobacilli in vitro (Hidalgo et al., 2012). Similar results were observed with a blueberry extract in mice (Molan et al., 2009) and powdered blueberry drink in humans (Vendrame et al., 2011). Furthermore, pomegranate extract improved symptoms in mice with DSS-induced colitis, which correlated with an increase in bifidobacteria, lactobacilli and members of the genus Clostridium (Larrosa et al., 2010). It is likely that an increased abundance of commensals upon polyphenol consumption limits the colonization and invasion of harmful or pathogenic bacteria (Larrosa et al., 2010). The reciprocal action between diet and gut microflora present a plausible mechanism of the anti-inflammatory potential of polyphenols.

\section{Bioavailability and stability of anthocyanins}

It is important to consider the metabolic fate of anthocyanins in the context of its anti-inflammatory activity in the gut. Anthocyanins are inherently unstable at $\mathrm{pH}>3.5$ and subject to spontaneous degradation in the gastrointestinal tract. Anthocyanins are metabolized by intestinal epithelial cells and conjugated by phase II enzymes upon absorption. Additionally, anthocyanins are readily catabolized by colonic microflora. Thus, anthocyanins are extensively transformed throughout the intestine (De Ferrars et al., 2014). It is widely accepted that due to poor bioavailability, and inherent instability that metabolites of anthocyanins may be responsible for any curative properties that anthocyanin rich foods may impart, rather than intact parent compounds (Fernandes et al., 2014; Wiczkowski et al., 2010).

\subsection{Stability and bioavailability of anthocyanins in the stom- ach and small intestine}

Anthocyanins are relatively stable in the stomach. The acidity in this region favors the flavylium ion and hemiketal forms of anthocyanins, which do not degrade readily (Fleschhut et al., 2006). Additionally, interaction with endogenous proteins and the food matrix serve to further stabilize these molecules (Correa-Betanzo et al., 2014; Fernandes et al., 2014). The half-life of anthocyanins in the stomach is estimated at 120 minutes (He et al., 2009). Because transit to the small intestines occurs in approximately 20-30 min, a large proportion of ingested anthocyanins reach the small intestine intact after ingestion.

Anthocyanins have been detected in the blood $30 \mathrm{~min}$ after aronia juice consumption, suggesting that anthocyanins are partially and specifically absorbed in the stomach tract (Wiczkowski et al., 2010). The absorption of intact anthocyanins by stomach cells has been established in vitro and was attributed to a saturable transport mechanism (Fernandes et al., 2012).

While anthocyanins can enter the blood stream via the stomach, small intestine, and colon, the bioavailability of these compounds is low. Several studies have found the bioavailability of anthocyanins to range from $0.4-2 \%$, which is quite low relative to other flavonoids (2.5-18.5\%) (Kay et al., 2005; Manach et al., 2005; Miyazawa and Nakagawa, 1999). Czank et al. (2013) established a much greater minimum relative bioavailability of $\mathrm{C}^{13}$-labeled $\mathrm{Cy}$ 3 Glu in humans of $12.4 \pm 1.4 \%$. The disparity in results may be a consequence of the administration of pure Cy3Glu as opposed to anthocyanins from a food matrix. The high interindividual variability of anthocyanin metabolism and bioavailability also play a role in studies of this type (Czank et al., 2013). Interindividual variability in anthocyanin metabolism and uptake has been well documented and is affected by intestinal enzyme activity, transit time, and gut microbes (He et al., 2009; Xie et al., 2016).

Glycosylation and acylation affect stability of anthocyanins in the upper GI tract. Acylation tends to impart stability to these molecules. Anthocyanin monoglucosides are more stable than corresponding aglycones, but further glycosylation reduces stability in diglucosides. For example, anthocyanins from blueberries were more readily recovered than those derived from pomegranates after in vitro simulated digestion (Correa-Betanzo et al., 2014; Pérez-Vicente, et al., 2002). This may be partially due to the greater quantity of 3-monoglucosides and 3,5-acylated anthocyanins present in blueberries (Correa-Betanzo et al., 2014; Pérez-Vicente et al., 2002)

It is estimated that 20 to $70 \%$ of anthocyanins pass through the small intestine intact, depending on interindividual variability, anthocyanin profile, food matrix, and sugar moiety (Bermúdez-Soto et al., 2007). When chyme enters the small intestine, pancreatic secretions increase luminal $\mathrm{pH}$ to 7.5-8.0 (Fernandes et al., 2014) Anthocyanins tend to form quinoid bases $(\mathrm{pH}=6-8)$ and chalcones $(\mathrm{pH}=7-8)$ at physiological $\mathrm{pH}$ resulting in a rapid decrease in stability of the parent anthocyanins (Ozdal et al., 2016) (Figure 3). Thus, anthocyanins are susceptible to ring fission yielding aldehydes and phenolic acids derived from the $\mathrm{A}$ and $\mathrm{B}$ rings of the parent anthocyanin (Fleschhut et al., 2006).

Anthocyanin glycosides can be enzymatically hydrolyzed during transit through the small intestine (Figure 4 ). The $\beta$-glucosidase, lactase phlorizin hydrolase (LPH) is produced in the brush border of the small intestinal cells and liberates aglycones from polyphenol glycosides (Dueñas et al., 2015). As the concentration of microbiota increases along the small intestine, microbial $\beta$-glucosidases can also significantly contribute to anthocyanin hydrolysis (Miyazawa and Nakagawa, 1999).

\subsection{Gastrointestinal absorption of anthocyanin glycosides}

Anthocyanins are absorbed to portal blood primarily by intestinal epithelial cells. It is estimated that $\sim 7 \%$ of the initial Cy3Glu anthocyanin dose can be absorbed in the small intestine (He et al., 2009; Wu et al., 2017; Yi et al., 2006). Glucose transporters, GLUT1 and GLUT3, as well as organic anion membrane carrier bilitranslocase contribute to the gastric absorption of intact anthocyanins (Oliveira et al., 2019; Passamonti et al., 2002). Active transporters such as GLUT2 and sodium-dependent glucose transporter (SGLT1) are responsible for transport of anthocyanin glycosides in intestinal epithelial cells (Faria et al., 2009; Wiczkowski et al., 2010). The type and orientation of glycosyl moieties of anthocyanins determine anthocyanin transport either through direct interaction with transporters or inhibition via stearic hindrance (Wiczkowski et al., 2010; Passamonti et al., 2002). Dietary glucose reduces anthocyanin excretion in vivo, most likely by saturating glucose transporters (Mülleder et al., 2002). Additionally, chronic 


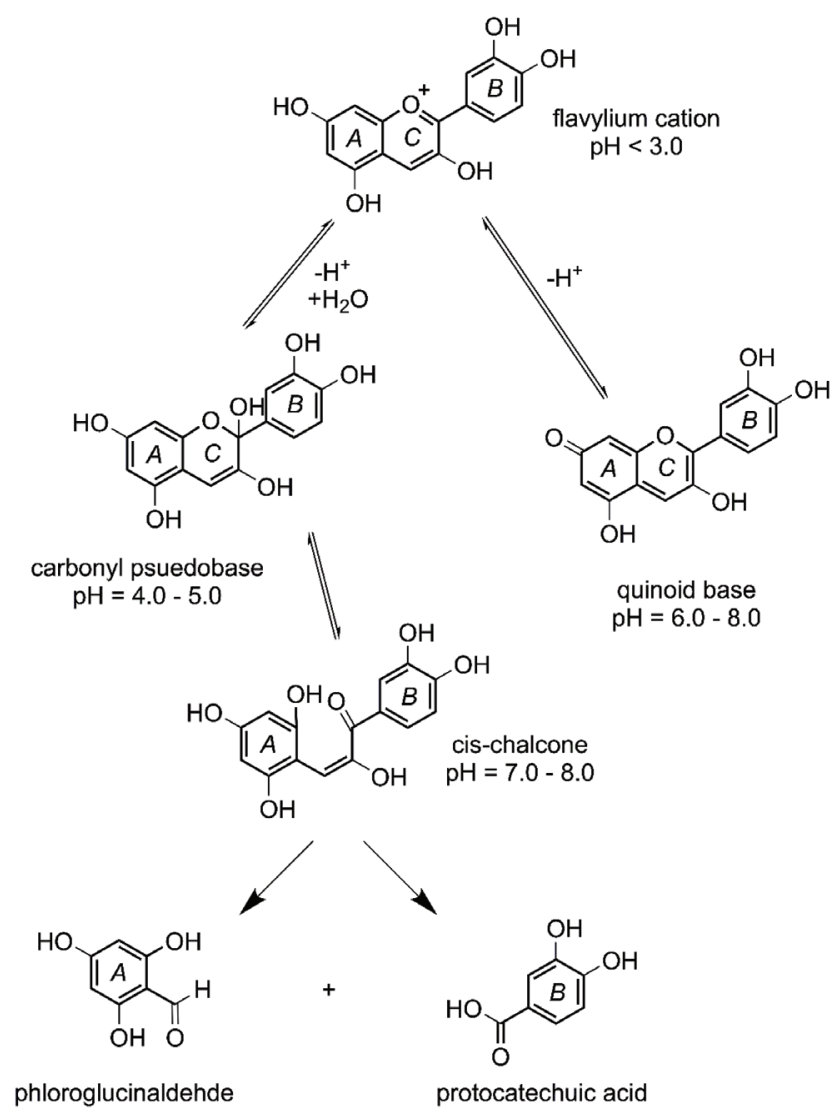

Figure 3. pH dependence of cyanidin and its degradation products. Adapted from: Fleschhut et al. (2006).

exposure to anthocyanins increases the expression of GLUT2 in Caco-2 cells (Faria et al., 2009). Therefore, food matrix and diet play a role in determining the bioavailability of anthocyanins.

\subsection{Biotransformation of anthocyanins}

Anthocyanins may be initially transformed by phase II enzymes in the epithelial cells. Glucuronidation, sulphation, and methylation occur in enterocytes (Fernandes et al., 2014; Kamiloglu et al., 2015). Subsequently, anthocyanins are distributed to the portal blood and are further metabolized by phase II enzymes in the liver. Hepatic phase II metabolism of polyphenols occurs via UDPglucuronosyl transferases, catechol-O-methyl transferases, sulfotransferases, and glutathione transferases (Rechner et al., 2004). Hepatocytes also catalyze hydrolysis and oxidation due to the ac- tion of cytochrome P450 and glucosidases (Rechner et al., 2004).

\subsection{Colonic anthocyanin metabolism}

After transit through the small intestine, the remaining anthocyanins enter the colon intact where they are readily catabolized by gut microbiota. Although $\sim 50 \%$ of the anthocyanins enter the large intestine, only $2-3 \%$ of are excreted in the feces (Correa-Betanzo et al., 2014). Although some anthocyanins are absorbed by colonic enterocytes, microbial catabolism accounts for the majority of colonic anthocyanin loss (Bermúdez-Soto et al., 2007).

The first step in microbial catabolism is the hydrolytic deglycosylation of the anthocyanin (Figure 4). The resulting anthocyanidin spontaneously degrades into phenolic acids and phloroglucinol aldehyde at the neutral to basic $\mathrm{pH}$ of the colon (Woodward et al., 2009). Anthocyanidins exist mainly as quinoid bases at $\mathrm{pH}=$ 8 , which form $\alpha$-diketone intermediates before scission of the adjoining C-ring (Fleschhut et al., 2006). Once liberated, the smaller phenolic acids can be readily absorbed by enterocytes, evidenced by the predominant appearance of catabolites after the parent anthocyanin maximum concentration $\left(\mathrm{C}_{\max }\right)$ in serum (Czank et al., 2013).

Enterohepatic recirculation promotes further microbial catabolism of anthocyanins. Anthocyanins and their metabolites are enriched in bile and the liver after ingestion (Felgines et al., 2010). Enterohepatic recirculation appears to be a primary elimination pathway for anthocyanin catabolites. For example, after Cy3Glu consumption, the primary compounds recovered in urine and serum during the first 6 hours were phase II metabolites of protocatechuic acid (PCA) (Czank et al., 2013). Anthocyanin biotransformation occurs rapidly, as PCA phase II metabolites have been detected in serum as early as 30 minutes after anthocyanin consumption (Czank et al., 2013). Furthermore, phase II metabolites of hippuric, phenylacetic, and phenylpropionic acid were the predominant anthocyanin catabolites observed after $24 \mathrm{~h}$ in serum (Czank et al., 2013). Considering the significance of enterohepatic recirculation in anthocyanin metabolism, it is important to consider that the ingested anthocyanin profile will be largely catabolized.

\subsubsection{Proposed metabolic pathways}

Considering the diversity of dietary polyphenols, the gut microbiota reduces a large number of parent polyphenolic compounds into a narrower range of catabolites (van Duynhoven et al., 2011). Cy3Glu is catabolized to benzoic acids, phenylpropionic acids, phenylacetic acids, benzaldehydes, PCA, coumaric acid, vanillic acids, caffeic acid, syringic acid and ferulic acid (Chen et al., 2017; Correa-Betanzo et al., 2014; Fleschhut et al., 2006; Xie et al., 2016). On the basis of these prior reports, the putative metabolic pathways of catabolites most abundant in the feces are depicted in

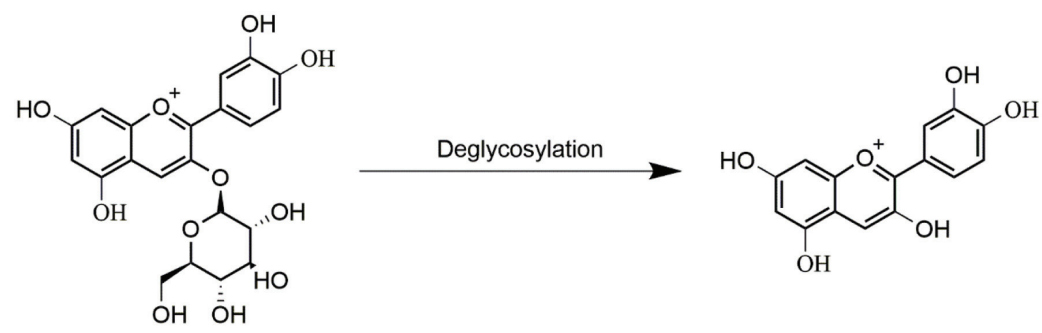

Figure 4. Deglycosylation of cyanidin-3-glucoside. 


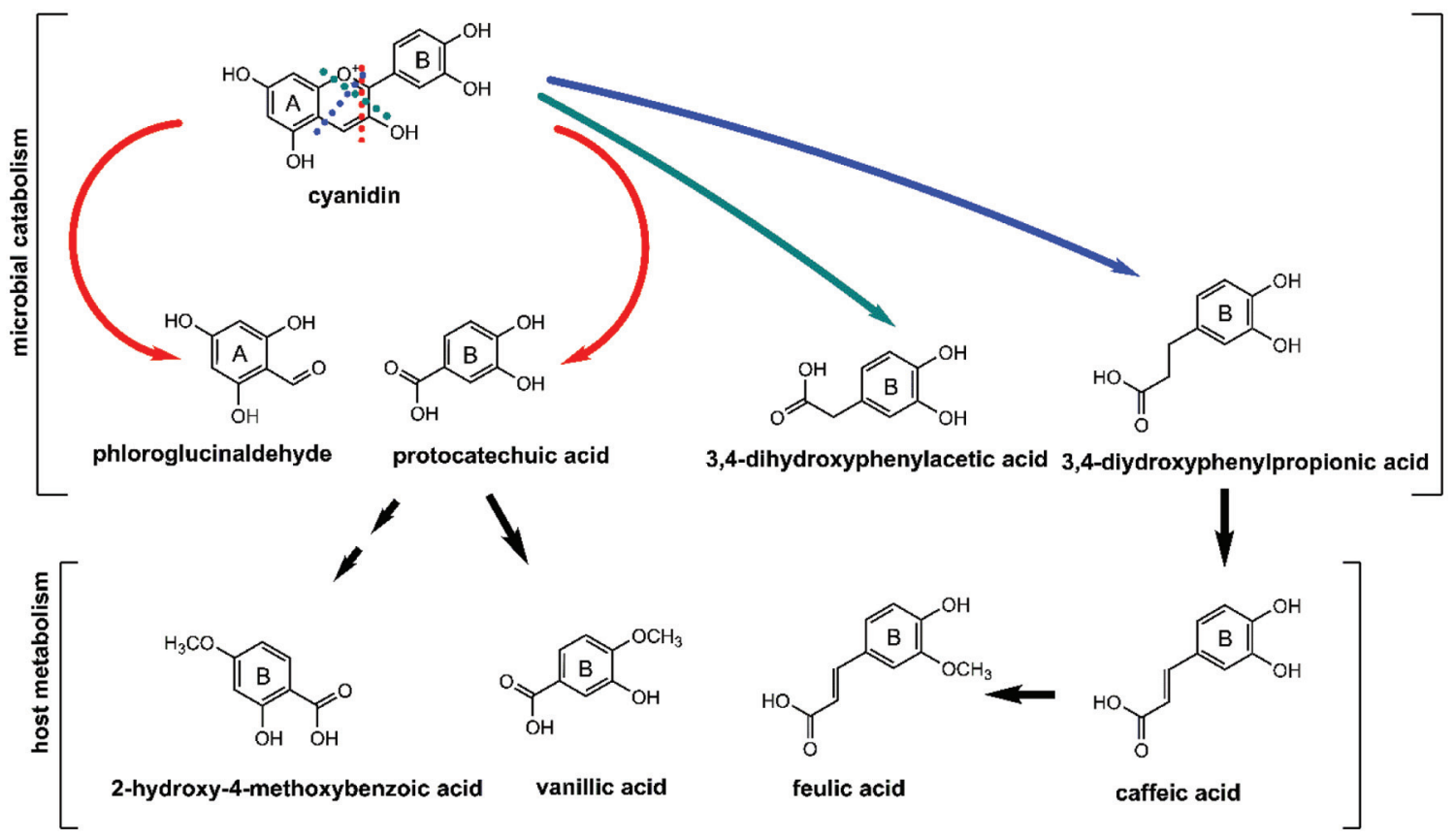

Figure 5. Colonic catabolites of cyanidin. Adapted from: De Ferrars et al. (2014), Fleschhut et al. (2006) and Xie et al. (2016).

Figure 5 (De Ferrars et al., 2014; González-Barrio et al., 2011; Xie et al., 2016). PCA was initially regarded as primary anthocyanin catabolite, but it is likely quickly subjected to further transformation. It is now recognized that the catabolic pool of anthocyanin varies by individual, food matrix, and background diet (De Ferrars et al., 2014).

\section{Bioactivity of anthocyanin catabolites}

Given the predominance of microbial-derived anthocyanin catabolites, it is important to consider the contribution of these compounds to bioactivity upon anthocyanin consumption. Pharmacokinetic and pre-clinical studies suggest a number of anthocyanin catabolites possess inherent anti-inflammatory activity, particularly in the intestine.

\subsection{Protocatechuic acid (PCA)}

Protocatechuic acid or 3,4-dihydroxy benzoic acid (3,4-DHBA) has been identified as the major degradation product of cyanidintype anthocyanins in several studies (Esposito et al., 2015; Felgines et al., 2003; Hanske et al., 2012; Vitaglione et al., 2007). A pharmacokinetic study in humans $(n=6)$ reported that consumption of blood orange juice $(71 \mathrm{mg} / \mathrm{L}$ cyanidin glucosides) resulted in recovery of $<2 \%$ of the parent anthocyanin in serum and urine, while the PCA catabolite pool accounted for $72.5 \%$ of the ingested cyanidin (Vitaglione et al., 2007). PCA is formed via scission of the cyanidin aglycone or dehydroxylation of dihydroxyphenylacetic acid (Vitaglione et al., 2007; Xie et al., 2016). Subsequently, PCA is rapidly transformed by metabolic processes, suggesting a high variability of exposure (De Ferrars et al., 2014; He et al., 2009).

PCA can directly inhibit intestinal inflammation. In a DSSinduced colitic rats, $10 \mathrm{mg} / \mathrm{kg}$ PCA added to the drinking water inhibited colitis-induced wasting, improved histopathology scores, decreased plasma IL-6, IL- $1 \beta$, and TNF- $\alpha$, and reduced colonic COX-2 and iNOS relative to the control (Farombi et al., 2016). Intraperitoneal injection of 30 or $60 \mathrm{mg} / \mathrm{kg}$ PCA in TNBS-induced colitic mice resulted in a similar global improvement of colitis progression and improvement in markers of oxidative stress including glutathione (GSH), superoxide dismutase (SOD), and catalase (Crespo et al., 2017). The suppression of TNBS-induced oxidative stress was attributed to elevated colonic Nrf2 in PCA-treated mice (Crespo et al., 2017). High conversion rates and bioactivity suggest PCA may contribute in-part to the anti-inflammatory activity of anthocyanin consumption.

\subsection{Ferulic acid (FA)}

Ferulic acid (FA or 4-hydroxy-3-methoxycinnamic acid) is a member of the hydroxycinnamic acid family of phenolics that is derived from the Cy3Glu A- or B-ring (Janicke et al., 2005). Ferulic acid can be formed by the rapid methylation of caffeic acid after absorption. FA was present in serum and urine within 4 hours of a $500 \mathrm{mg} 6,8,10,3^{\prime}, 5^{\prime}-{ }^{13} \mathrm{C} 5$-Cy3 Glu bolus dose in healthy humans (De Ferrars et al., 2014). Colonic epithelial transport of FA (freeform and conjugated) via a monocarboxylate, multidrug resistance protein, and P-glycoprotein transporters was observed in Caco-2 cells as well as ex vitro in rat everted colonic sacs (Poquet et al., 2008). FA is abundant in the feces after anthocyanin consumption, a result of multiple convergent pathways and a relatively long halflife (De Ferrars et al., 2014).

FA also inhibits colitis in rodents. Intrarectal administration of FA (20 and $40 \mathrm{mg} / \mathrm{kg}$ ) in a mouse model of TNBS-induced colitis 14 days after challenge reduced disease severity (Sadar et al., 2016). FA significantly improved histopathological indices of inflammation, protected intestinal mucosal cells from apoptosis, and reduced colonic oxidative stress (Sadar et al., 2016). FA administration also inhibited the pro-inflammatory cytokines IL-1 $\beta$, IL-6, and TNF- $\alpha$ and increased immunoregulatory IL-10 (Sadar et al., 
2016).

FA appears to inhibit colitis via a mechanism similar to 5-aminosalicylic acid, a metabolite of common IBD drug, sulfasalazine. Administration of intrarectal sodium ferulate at $800 \mathrm{mg} / \mathrm{kg}$ b.w. was as effective as $100 \mathrm{mg} / \mathrm{kg}$ b.w. of 5 -aminosalicylic acid in preventing colonic mucosal injury due to acetic acid-induced colitis in Sprague-Dawley rats (Dong et al., 2003). FA consumption (20 and $40 \mathrm{mg} / \mathrm{kg}$ ) also inhibited acetic acid induced colitis in rats via modulation of the production of cellular antioxidants, specifically superoxide dismutase (SOD) and glutathione (GSH), via an Nrf2dependent pathway (Kandhare et al., 2016).

The anti-inflammatory effects of FA administration can also modulate intestinal barrier function in cultured cells. Caco- 2 cells pretreated with ferulic acid $(5$ or $15 \mu \mathrm{M})$ resisted the reduction in TEER from a TNBS challenge (He et al., 2018). FA improved intestinal permeability by reducing apoptosis and increasing the tight junction proteins ZO-1 and occludin (He et al., 2018). Due to the oxidative damage caused by TNBS, it was suggested that the apparent effect of FA on barrier function was due to either direct or indirect antioxidant function (He et al., 2018).

\subsection{Vanillic acid (VA)}

Vanillic acid (VA or 4-hydroxy-3-methoxy benzoic acid) is formed from PCA by host catechol-O-methyltransferase, or from FA by $\beta$-oxidation by the gut microbiota (Huang et al., 1993). VA is the primary initial B-ring fragment of peonidin, a methylated metabolite of cyanidins (Ozdal et al., 2016; Xie et al., 2016). VA can be formed from Cy3Glu in both gnotobiotic and human-conventionalized rat, suggesting that both human and microbial metabolism play a role in the formation of this compound (Hanske et al., 2012).

In humans, VA was a predominate catabolite in the serum after ingestion of $500 \mathrm{mg}$ of ${ }^{13} \mathrm{C} 5-\mathrm{Cy} 3 \mathrm{Glu}$, however its conversion rate was highly variable and only detected in two of eight healthy male subjects (De Ferrars et al., 2014). In contrast, in the same study, VA was the primary metabolite recovered in urine mainly as methylated and sulfated conjugates. VA thus appears to be an important intermediate in the biotransformation involved in anthocyanin clearance (De Ferrars et al., 2014).

VA also possesses intrinsic anti-inflammatory activity. VA inhibited NF- $\mathrm{KB}$ activation and suppressed the phosphorylation of MAPK in the human mast cell line HMC-1 (Jeong et al., 2017). VA consumption inhibited DSS-induced colitis in mice and reduced IL-6, COX-2, and NF- $\kappa$ B activation in colon tissue (Kim et al., 2010). Additionally, the analgesic and anti-inflammatory potential of the phenolic compound was demonstrated in chemically-induced (acetic acid, phenyl-p-benzoquinone, carrageenan, formalin, and complete Freund's adjuvant) paw edema in mice (Calixto-Campos et al., 2015). NF-kB-induced cytokine production, and activation of cellular antioxidant system were inhibited by VA (Calixto-Campos et al., 2015). Similar to PCA and FA, VA may also contribute the anti-inflammatory effects of anthocyanin consumption in the gut.

\section{Conclusion}

Consumption of anthocyanins and anthocyanin-rich berries consistently inhibit IBD in rodent models of colitis. Although data from human intervention studies is limited, additional human intervention studies for inhibiting IBD with anthocyanin-rich berries are warranted. Intestinal barrier function is a central factor in maintaining chronic inflammation in IBD. Anthocyanins and anthocyanin metabolites inhibit colitis and improve intestinal barrier function by a number of interconnected mechanisms. These bioactives modulate oxidative stress and inflammation directly in intestinal epithelial cells, but also modulate gut microbiota, affect activation of immune cells, and modulate cytokine activity.

Despite the promising nature of anthocyanins for IBD, a number of gaps in knowledge remain. The distribution and catabolic fate of anthocyanins has significant variability between individuals and the food matrix. It is currently unclear how the inter-individual variability in anthocyanin catabolism affects anti-colitic efficacy. The food matrix also is expected to affect the distribution of anthocyanin and catabolites in the colon. Experiments utilizing gnotobiotic rodents colonized with defined microbial communities could help resolve these gaps. However, the differences in efficacy between juice, whole fruit, and anthocyanin-rich berry extracts remains unresolved. Efforts to resolve these questions utilizing rodent and cell models should consider the metabolic fate of anthocyanins to maximize translation to human intervention studies using berry bioactives for IBD.

\section{Acknowledgments}

BWB supported by USDA NIFA WIS01909, JCV supported by a fellowship from the SciMed Graduate Research Scholars program at the University of Wisconsin-Madison.

\section{References}

Abraham, C., Dulai, P.S., Vermeire, S., and Sandborn, W.J. (2017). Lessons learned from trials targeting cytokine pathways in patients with inflammatory bowel diseases. Gastroenterology 152(2): 374-388.e4.

Ahluwalia, B., Moraes, L., Magnusson, M.K., and Öhman, L. (2018). Immunopathogenesis of inflammatory bowel disease and mechanisms of biological therapies. Scand. J. Gastroenterol. 53(4): 379-389.

Bamforth, S.D., Kniesel, U., Wolburg, H., Engelhardt, B., and Risau, W. (1999). A dominant mutant of occludin disrupts tight junction structure and function. J. Cell Sci. 112(12): 1879-1888.

Banan, A., Choudhary, S., Zhang, Y., Fields, J.Z., and Keshavarzian, A. (1999). Ethanol-induced barrier dysfunction and its prevention by growth factors in human intestinal monolayers: evidence for oxidative and cytoskeletal mechanisms. J. Pharmacol. Exp. Ther. 291(3): 1075-1085.

Basuroy, S., Seth, A., Elias, B., Naren, A.P., and Rao, R. (2006). MAPK interacts with occludin and mediates EGF-induced prevention of tight junction disruption by hydrogen peroxide. Biochem. J. 393(1): 69-77.

Bayele, H.K., Debnam, E.S., and Srai, K.S. (2016). Nrf2 transcriptional derepression from Keap1 by dietary polyphenols. Biochem. Biophys. Res. Commun. 469(3): 521-528.

Bermúdez-Soto, M.J., Tomás-Barberán, F.A., and García-Conesa, M.T. (2007). Stability of polyphenols in chokeberry (Aronia melanocarpa) subjected to in vitro gastric and pancreatic digestion. Food Chem. 102(3): 865-874.

Biedermann, L., Mwinyi, J., Scharl, M., Frei, P., Zeitz, J., Kullak-Ublick, G.A., Vavricka, S.R., Fried, M., Weber, A., Humpf, H.U., Peschke, S., Jetter, A., Krammer, G., and Rogler, G. (2013). Bilberry ingestion improves disease activity in mild to moderate ulcerative colitis - an open pilot study. J. Crohns. Colitis 7(4): 271-279.

Blair, S.A., Kane, S.V., Clayburgh, D.R., and Turner, J.R. (2006). Epithelial myosin light chain kinase expression and activity are upregulated in inflammatory bowel disease. Lab. Investig. 86: 191-201.

Broom, O.J., Widjaya, B., Troelsen, J., Olsen, J., and Nielsen, O.H. (2009). Mitogen activated protein kinases: a role in inflammatory bowel disease? Clin. Exp. Immunol. 158(3): 272-280.

Bruewer, M., Utech, M., Ivanov, A.I., Hopkins, A.M., Parkos, C.A., and Nus- 
rat, A. (2005). Interferon- $\gamma$ induces internalization of epithelial tight junction proteins via a macropinocytosis-like process. FASEB J. 19(8): 923-933.

Calixto-Campos, C., Carvalho, T.T., Hohmann, M.S.N., Pinho-Ribeiro, F.A. Fattori, V., Manchope, M.F., Zarpelon, A.C., Baracat, M.M., Georgetti, S.R., Casagrande, R., and Verri, W.A. (2015). Vanillic acid inhibits inflammatory pain by inhibiting neutrophil recruitment, oxidative stress, cytokine production, and NFKB activation in mice. J. Nat. Prod. 78(8): 1799-1808.

Chen, Y., Li, Q., Zhao, T., Zhang, Z., Mao, G., Feng, W., Wu, X., and Yang, L. (2017). Biotransformation and metabolism of three mulberry anthocyanin monomers by rat gut microflora. Food Chem. 237: 887-894.

Chiba, H., Osanai, M., Murata, M., Kojima, T., and Sawada, N. (2008). Transmembrane proteins of tight junctions. Biochim. Biophys. Acta - Biomembr. 1778(3): 588-600.

Cohen, A.B., Lee, D., Long, M.D., Kappelman, M.D., Martin, C.F., Sandler R.S., and Lewis, J.D. (2013). Dietary patterns and self-reported associations of diet with symptoms of inflammatory bowel disease. Dig. Dis. Sci. 58(5): 1322-1328.

Correa-Betanzo, J., Allen-Vercoe, E., McDonald, J., Schroeter, K., Corredig, M., and Paliyath, G. (2014). Stability and biological activity of wild blueberry (Vaccinium angustifolium) polyphenols during simulated in vitro gastrointestinal digestion. Food Chem. 165: 522-531.

Crespo, I., San-Miguel, B., Mauriz, J.L., Ortiz de Urbina, J.J., Almar, M., Tuñón, M.J., and González-Gallego, J. (2017). Protective effect of protocatechuic acid on TNBS-induced colitis in mice is associated with modulation of the SphK/S1P signaling pathway. Nutrients 9(3): 288.

Czank, C., Cassidy, A., Zhang, Q., Morrison, D.J., Preston, T., Kroon, P.A., Botting, N.P., and Kay, C.D. (2013). Human metabolism and elimination of the anthocyanin, cyanidin-3-glucoside: a 13 C-tracer study. Am. J. Clin. Nutr. 97: 995-1003.

De Ferrars, R.M., Czank, C., Zhang, Q., Botting, N.P., Kroon, P.A., Cassidy, A., and Kay, C.D. (2014). The pharmacokinetics of anthocyanins and their metabolites in humans. Br. J. Pharmacol. 171(13): 3268-3682.

Deiana, M., Loru, D., Incani, A., Rosa, A., Atzeri, A., Melis, M.P., and et al (2012). Wine extracts from Sardinian grape varieties attenuate membrane oxidative damage in Caco-2 cell monolayers. Food Chem. 134(4): 2105-2113.

Dong, W.-G., Liu, S.-P., Yu, B.-P., Wu, D.-F., Luo, H.-S., and Yu, J.-P. (2003). Ameliorative effects of sodium ferulate on experimental colitis and their mechanisms in rats. World J. Gastroenterol. 9(11): 2533-2538.

Dueñas, M., Muñoz-González, I., Cueva, C., Jiménez-Girón, A., SánchezPatán, F., Santos-Buelga, C., Moreno-Arribas, M.V., and Bartolomé, B. (2015). A survey of modulation of gut microbiota by dietary polyphenols. Biomed Res. Int. 2015: 850902.

Esposito, D., Damsud, T., Wilson, M., Grace, M.H., Strauch, R., Li, X., Lila, M.A., and Komarnytsky, S. (2015). Black currant anthocyanins attenuate weight gain and improve glucose metabolism in diet-induced obese mice with intact, but not disrupted, gut microbiome. J. Agric. Food Chem. 63(27): 6172-6180.

Fanning, A.S., Anderson, J.M., Saltiel, A.R., and Margolis, B.L. (1999). PDZ domains: fundamental building blocks in the organization of protein complexes at the plasma membrane. J. Clin. Invest. 103(6): 767-772.

Faria, A., Pestana, D., Azevedo, J., Martel, F., Freitas, V.d.e., Azevedo, I., Mateus, N., and Calhau, C. (2009). Absorption of anthocyanins through intestinal epithelial cells - putative involvement of GLUT2. Mol. Nutr. Food Res. 53(11): 1430-1437.

Farombi, E.O., Adedara, I.A., Awoyemi, O.V., Njoku, C.R., Micah, G.O., Esogwa, C.U., Owumi, S.E., and Olopade, J.O. (2016). Dietary protocatechuic acid ameliorates dextran sulphate sodium-induced ulcerative colitis and hepatotoxicity in rats. Food Funct. 7(2): 913-921.

Felgines, C., Krisa, S., Mauray, A., Besson, C., Lamaison, J.L., Scalbert, A., Mérillon, J.M., and Texier, O. (2010). Radiolabelled cyanidin 3-O-glucoside is poorly absorbed in the mouse. Br. J. Nutr. 103(12): 17381745.

Felgines, C., Verine, S., Ra, T., Gonthier, M.P., Texier, O., Scalbert, A., Lamaison, J.L., and Rémésy, C. (2003). Human nutrition and metabolism strawberry anthocyanins are recovered in urine as glucuro- and sulfoconjugates in humans. J. Nutr. 133: 1296-1301.

Feng, Y.J., and Li, Y.Y. (2011). The role of p38 mitogen-activated protein kinase in the pathogenesis of inflammatory bowel disease. J. Dig. Dis.
12(5): 327-332

Fernandes, I., de Freitas, V., Reis, C., and Mateus, N. (2012). A new approach on the gastric absorption of anthocyanins. Food Funct. 3(5): 508-516.

Fernandes, I., Faria, A., Calhau, C., de Freitas, V., and Mateus, N. (2014). Bioavailability of anthocyanins and derivatives. J. Funct. Foods $7(1)$ : 54-66.

Ferrari, D., Speciale, A., Cristani, M., Fratantonio, D., Molonia, M.S., Ranaldi, G., Saija, A., and Cimino, F. (2016). Cyanidin-3-O-glucoside inhibits NF-kB signalling in intestinal epithelial cells exposed to TNF- $\alpha$ and exerts protective effects via Nrf2 pathway activation. Toxicol. Lett. 264: 51-58.

Fish, S.M., Proujansky, R., and Reenstra, W.W. (1999). Synergistic effects of interferon $\gamma$ and tumour necrosis factor $\alpha$ on T84 cell function. Gut 45(2): 191-198.

Fleschhut, J., Kratzer, F., Rechkemmer, G., and Kulling, S.E. (2006). Stability and biotransformation of various dietary anthocyanins in vitro. Eur. J. Nutr. 45(1): 7-18.

Fujita, H., Chiba, H., Yokozaki, H., Sakai, N., Sugimoto, K., Wada, T., Kojima, T., Yamashita, T., and Sawada, N. (2006). Differential expression and subcellular localization of claudin-7,-8,-12,-13, and-15 along the mouse intestine. J. Histochem. Cytochem. 54(8): 933-944.

Furuse, M. (2010). Molecular basis of the core structure of tight junctions. Cold Spring Harb. Perspect. Biol. 2(1): a002907.

Garcia-Hernandez, V., Quiros, M., and Nusrat, A. (2017). Intestinal epithelial claudins: expression and regulation in homeostasis and inflammation. Annu. New York Acad. Sci. 1397(1): 66-79.

Gersemann, M., Wehkamp, J., Fellermann, K., and Stange, E.F. (2008). Crohn's disease - defect in innate defence. World J. Gastroenterol. 14(36): 5499-5503.

Gerstgrasser, A., Melhem, H., Leonardi, I., Atrott, K., Schäfer, M., Werner, S., Rogler, G., and Frey-Wagner, I. (2017). Cell-specific activation of the Nrf2 antioxidant pathway increases mucosal inflammation in acute but not in chronic colitis. J. Crohn's Colitis 11(4): 485-499.

González-Barrio, R., Edwards, C.A., and Crozier, A. (2011). Colonic catabolism of ellagitannins, ellagic acid, and raspberry anthocyanins: In vivo and in vitro studies. Drug Metab. Dispos. 39(9): 1680-1688.

Graham, W.V., Wang, F., Clayburgh, D.R., Cheng, J.X., Yoon, B., Wang, Y., Lin, A., and Turner, J.R. (2006). Tumor Necrosis factor-induced long myosin light chain kinase transcription is regulated by differentiation-dependent signaling events: characterization of the human long myosin light chain kinase promoter. J. Biol. Chem. 281(36): 26205-26215.

Hanske, L., Engst, W., Loh, G., Sczesny, S., Blaut, M., and Braune, A. (2012). Contribution of gut bacteria to the metabolism of cyanidin 3-glucoside in human microbiota-associated rats. Br. J. Nutr. 109(8): 14331441.

He, J., Wallace, T.C., Keatley, K.E., Failla, M.L., and Giusti, M.M. (2009). Stability of black raspberry anthocyanins in the digestive tract lumen and transport efficiency into gastric and small intestinal tissues in the rat. J. Agric. Food Chem. 57(8): 3141-3148.

He, S., Guo, Y., Zhao, J., Xu, X., Song, J., Wang, N., and Liu, Q. (2018). Ferulic acid protects against heat stress-induced intestinal epithelial barrie dysfunction in IEC- 6 cells via the PI3K/Akt-mediated Nrf2/HO-1 signaling pathway. Int. J. Hyperth. 35(1): 112-121.

Hidalgo, M., Oruna-Concha, M.J., Kolida, S., Walton, G.E., Kallithraka, S. Spencer, J.P.E., and De Pascual-Teresa, S. (2012). Metabolism of anthocyanins by human gut microflora and their influence on gut bacterial growth. J. Agric. Food Chem. 60(15): 3882-3890.

Himes, S.R., Sester, D.P., Ravasi, T., Cronau, S.L., Sasmono, T., and Hume, D.A. (2006). The JNK are important for development and survival of macrophages. J. Immunol. 176(4): 2219-2228.

Hollman, P.C.H., Cassidy, A., Comte, B., Heinonen, M., Richelle, M., Richling, E., Serafini, M., Scalbert, A., Sies, H., and Vidry, S. (2011). The biological relevance of direct antioxidant effects of polyphenols for cardiovascular health in humans is not established. J. Nutr. 141(5): 989S-1009S.

Huang, Z., Dostal, L., and Rosazzas, J.P.N. (1993). Mechanisms of ferulic acid conversions to vanillic acid and guaiacol by Rhodotorula rubra. J. Biol. Chem. 268(32): 23954-23958.

Janicke, B., Onning, G., and Oredsson, S.M. (2005). Differential effects of ferulic acid and p-coumaric acid on $\mathrm{S}$ phase distribution and length 
of $\mathrm{S}$ phase in the human colonic cell line Caco-2. J. Agric. Food Chem. 53(17): 6658-6665.

Jeong, H.-J., Nam, S.-Y., Kim, H.-Y., Jin, M.H., Kim, M.H., Roh, S.S., and Kim, H.-M. (2017). Anti-allergic inflammatory effect of vanillic acid through regulating thymic stromal lymphopoietin secretion from activated mast cells. Nat. Prod. Res. Oct. 16: 1-5.

Jin, W., Wang, H., Ji, Y., Hu, Q., Yan, W., Chen, G., and Yin, H. (2008). Increased intestinal inflammatory response and gut barrier dysfunction in Nrf2-deficient mice after traumatic brain injury. Cytokine 44(1): 135-140.

Jung, H., Lee, H.J., Cho, H., and Hwang, K.T. (2015). Anti-inflammatory activities of Rubus fruit anthocyanins in inflamed human intestinal epithelial cells. J. Food Biochem. 39(3): 300-309.

Kamiloglu, S., Capanoglu, E., Grootaert, C., and van Camp, J. (2015). Anthocyanin absorption and metabolism by human intestinal Caco-2 cells-a review. Int. J. Mol. Sci. 16(9): 21555-21574.

Kandhare, A.D., Patil, A., Guru, A., Mukhrjee, A., Sarkar, S., Sengupta, A., Parmar, H.M., Muthal, A.P., Wangikar, P., and Bodhankar, S.L. (2016). Ameliorative effect of ferulic acid against acetic acid induced ulcerative colitis: role of HO-1 and Nrf2. Parmacologia 7(2): 114-124.

Kay, C.D., Mazza, G., and Holub, B.J. (2005). Human nutrition and metabolism anthocyanins exist in the circulation primarily as metabolites in adult men. J. Nutr. 135: 2582-2588.

Kim, H., Banerjee, N., Sirven, M.A., Minamoto, Y., Markel, M.E., Suchodolski, J.S., Talcot, S.T., and Mertens-Talcott, S.U. (2017). Pomegranate polyphenolics reduce inflammation and ulceration in intestinal colitis-involvement of the miR-145/p70S6K1/HIF1 $\alpha$ axis in vivo and in vitro. J. Nutr. Biochem. 43: 107-115.

Kim, S.J., Kim, M.C., Um, J.Y., and Hong, S.H. (2010). The beneficial effect of vanillic acid on ulcerative colitis. Molecules 15(10): 7208-7217.

Kimura, K., Teranishi, S., and Nishida, T. (2009). Interleukin-1 $\beta$-induced disruption of barrier function in cultured human corneal epithelial cells. Invest. Ophthalmol. Vis. Sci. 50(2): 597-603.

Kucharzik, T., LÜgering, N., Pauels, H.-G., Domschke, W., and Stoll, R. (1998). IL-4, IL-10 and IL-13 down-regulate monocyte-chemoattracting protein-1 (MCP-1) production in activated intestinal epithelial cells. Clin. Exp. Immunol. 111(1): 152-157.

Kuntz, S., Asseburg, H., Dold, S., Römpp, A., Fröhling, B., Kunz, C., and Rudloff, S. (2015). Inhibition of low-grade inflammation by anthocyanins from grape extract in an in vitro epithelial-endothelial co-culture model. Food Funct. 6(4): 1136-1149.

Lane, E.R., Zisman, T.L., and Suskind, D.L. (2017). The microbiota in inflammatory bowel disease: current and therapeutic insights. J. Inflamm. Res. 10: 63-73.

Larrosa, M., González-Sarrías, A., Yáñez-Gascón, M.J., Selma, M.V., AzorínOrtuño, M., Toti, S., Tomás-Barberán, F., Dolara, P., and Espín, J.C. (2010). Anti-inflammatory properties of a pomegranate extract and its metabolite urolithin-A in a colitis rat model and the effect of colon inflammation on phenolic metabolism. J. Nutr. Biochem. 21(8): 717-725.

Le Chatelier, E., Nielsen, T., Qin, J., Prifti, E., Hildebrand, F., Falony, G., and et al (2013). Richness of human gut microbiome correlates with metabolic markers. Nature 500(7464): 541-546.

Lee, S.H. (2015). Intestinal permeability regulation by tight junction: implication on inflammatory bowel diseases. Intest. Res. 13(1): 11-18.

Li, L., Wang, L., Wu, Z., Yao, L., Wu, Y., Huang, L., Liu, K., Zhou, X., and Gou, D. (2014). Anthocyanin-rich fractions from red raspberries attenuate inflammation in both RAW264.7 macrophages and a mouse model of colitis. Sci. Rep. 4: 6234

Liang, G.H., and Weber, C.R. (2014). Molecular aspects of tight junction barrier function. Curr. Opin. Pharmacol. 19: 84-89.

Lingaraju, A., Long, T.M., Wang, Y., Austin, J.R., and Turner, J.R. (2015). Conceptual barriers to understanding physical barriers. Semin. Cell Dev. Biol. 42: 13-21.

Liu, C., Zhu, L., Fukuda, K., Ouyang, S., Chen, X., Wang, C., Zhang, C.J., Martin, B., Gu, C., Qin, L., Rachakonda, S., Aronica, M., Qin, J., and $\mathrm{Li}, \mathrm{X}$. (2017). The flavonoid cyanidin blocks binding of the cytokine interleukin-17A to the IL-17RA subunit to alleviate inflammation in vivo. Sci. Signal. 10(467): eaaf8823.

Ma, T.Y., Iwamoto, G.K., Hoa, N.T., Akotia, V., Pedram, A., Boivin, M.A., and Said, H.M. (2004). TNF- $\alpha$-induced increase in intestinal epithelial tight junction permeability requires NF-KB activation. Am. J. Phsiol. Gastrointest. Liver Physiol. 286(3): G367-376.

Manach, C., Williamson, G., Morand, C., Scalbert, A., and Rémésy, C. (2005). Bioavailability and bioefficacy of polyphenols in humans. I. Review of 97 bioavailability studies. Am. J. Clin. Nutr. 81(1): 230S242S.

Manichanh, C., Rigottier-Gois, L., Bonnaud, E., Gloux, K., Pelletier, E., Frangeul, L., Nalin, R., Jarrin, C., Chardon, P., Marteau, P., Roca, J., and Dore, J. (2006). Reduced diversity of faecal microbiota in Crohn's disease revealed by a metagenomic approach. Gut 55(2): 205-211.

Marlow, G.J., van Gent, D., and Ferguson, L.R. (2013). Why interleukin-10 supplementation does not work in Crohn's disease patients. World J. Gastroenterol. 19(25): 3931-3941.

Martin, D.A., and Bolling, B.W. (2015). A review of the efficacy of dietary polyphenols in experimental models of inflammatory bowel diseases. Food Funct. 6(6): 1773-1786.

Michielan, A., and D'Incà, R. (2015). Intestinal permeability in inflammatory bowel disease: pathogenesis, clinical evaluation, and therapy of leaky gut. Mediators Inflamm. 2015: 628157.

Miyazawa, T., and Nakagawa, K. (1999). Direct intestinal absorption of red fruit anthocyanins, cyanidin-3-glucoside and cyanidin-3, 5-diglucoside, into rats and humans. J. Agric. Food Chem. 47(3): 1083-1091.

Molan, A.L., Lila, M.A., Mawson, J., and De, S. (2009). In vitro and in vivo evaluation of the prebiotic activity of water-soluble blueberry extracts. World J. Microbiol. Biotechnol. 25(7): 1243-1249.

Morgan, X.C., Tickle, T.L., Sokol, H., Gevers, D., Devaney, K.L., Ward, D.V. and et al (2012). Dysfunction of the intestinal microbiome in inflammatory bowel disease and treatment. Genome Biol. 13(9): R79.

Mosele, J.I., Macià, A., and Motilva, M.J. (2015). Metabolic and microbial modulation of the large intestine ecosystem by non-absorbed diet phenolic compounds: a review. Molecules 20(9): 17429-17468.

Mülleder, U., Murkovic, M., and Pfannhauser, W. (2002). Urinary excretion of cyanidin glycosides. J. Biochem. Biophys. Methods. 53(1-3): 61-66.

Murphy, L.Y.W., Ling, K.H., El-Nezamia, H., and Wanga, M. (2018). Oxyresveratrol protective effects against deoxynivalenol-induced intestinal barrier dysfunction and bacterial translocation on porcine intestinal epithelial IPEC-J2 cells. J. Food Bioactives 1: 2-9.

Nemeth, Z.H., Bogdanovski, D.A., Barratt-Stopper, P., Paglinco, S.R., Antonioli, L., and Rolandelli, R.H. (2017). Crohn's Disease and ulcerative colitis show unique cytokine profiles. Cureus 9(4): e1177.

Nunes, C., Ferreira, E., Freitas, V., Almeida, L., Barbosa, R.M., and Laranjinha, J. (2013). Intestinal anti-inflammatory activity of red wine extract: unveiling the mechanisms in colonic epithelial cells. Food Funct. 4(3): 373-383.

Oliveira, H., Roma-Rodrigues, C., Santos, A., Veigas, B., Brás, N., Faria, A., and et al (2019). GLUT1 and GLUT3 involvement in anthocyanin gastric transport - nanobased targeted approach. Sci. Rep. 9(1): 789.

Ott, S.J., Musfeldt, M., Wenderoth, D.F., Hampe, J., Brant, O., Fölsch, U.R., Timmis, K.N., and Schreiber, S. (2004). Reduction in diversity of the colonic mucosa associated bacterial microflora in patients with active inflammatory bowel disease. Gut 53(5): 685-693.

Ozdal, T., Sela, D.A., Xiao, J., Boyacioglu, D., Chen, F., and Capanoglu, E. (2016). The reciprocal interactions between polyphenols and gut microbiota and effects on bioaccessibility. Nutrients 8(2): 78.

Passamonti, S., Vrhovsek, U., and Mattivi, F. (2002). The interaction of anthocyanins with bilitranslocase. Biochem. Biophys. Res. Commun. 296(3): 631-636

Pérez-Vicente, A., Gil-Izquierdo, A., and García-Viguera, C. (2002). In vitro gastrointestinal digestion study of pomegranate juice phenolic compounds, anthocyanins, and vitamin C. J. Agric. Food Chem. 50(8): 2308-2312.

Pervin, M., Hasnat, M.A., Lim, J.H., Lee, Y.M., Kim, E.O., Um, B.H., and Lim, B.O. (2016). Preventive and therapeutic effects of blueberry (Vaccinium corymbosum) extract against DSS-induced ulcerative colitis by regulation of antioxidant and inflammatory mediators. J. Nutr. Biochem. 28: 103-113.

Poquet, L., Clifford, M.N., and Williamson, G. (2008). Transport and metabolism of ferulic acid through the colonic epithelium. Drug Met. Disp. 36(1): 190-197.

Raleigh, D.R., Boe, D.M., Yu, D., Weber, C.R., Marchiando, A.M., Bradford, 
E.M., and et al (2011). Occludin S408 phosphorylation regulates tight junction protein interactions and barrier function. J. Cell Biol. 193(3): 565-582.

Rao, R., Baker, R.D., and Baker, S.S. (1999). Inhibition of oxidant-induced barrier disruption and protein tyrosine phosphorylation in Caco-2 cell monolayers by epidermal growth factor. Biochem. Pharmacol. 57(6): 685-695.

Rechner, A.R., Smith, M.A., Kuhnle, G., Gibson, G.R., Debnam, E.S., Srai, S.K.S., Moore, K.P., and Rice-Evans, C.A. (2004). Colonic metabolism of dietary polyphenols: Influence of structure on microbial fermentation products. Free Radic. Biol. Med. 36(2): 212-225.

Romier-Crouzet, B., Van De Walle, J., During, A., Joly, A., Rousseau, C., Henry, O., Larondelle, Y., and Schneider, Y.J. (2009). Inhibition of inflammatory mediators by polyphenolic plant extracts in human intestinal Caco-2 cells. Food Chem. Toxicol. 47(6): 1221-1230.

Romier, B., Van De Walle, J., During, A., Larondelle, Y., and Schneider, Y.J. (2008). Modulation of signalling nuclear factor-kappaB activation pathway by polyphenols in human intestinal Caco- 2 cells. Br. J. Nutr. 100(3): 542-551.

Roth, S., Spalinger, M.R., Gottier, C., Biedermann, L., Zeitz, J., Lang, S., Weber, A., Rogler, G., and Scharl, M. (2016). Bilberry-derived anthocyanins modulate cytokine expression in the intestine of patients with ulcerative colitis. PLoS One 11(5): 1-17.

Rothwell, J.A., Perez-Jimenez, J., Neveu, V., Medina-Ramon, A., M'Hiri, N., Carcia Lobato, P., and et al (2013). Phenol-Explorer 3.0: a major update of the Phenol-Explorer database to incorporate data on the effects of food processing on polyphenol content. Database 2013: bat070.

Ruffer, C., and Gerke, V. (2004). The C-terminal cytoplasmic tail of claudins 1 and 5 but not its PDZ-binding motif is required for apical localization at epithelial and endothelial tight junctions. Eur. J. Cell Biol. 83: 135-144.

Sadar, S.S., Vyawahare, N.S., and Bodhankar, S.L. (2016). Ferulic acid ameliorates tnbs-induced ulcerative colitis through modulation of cytokines, oxidative stress, iNos, Cox-2, and apoptosis in laboratory rats. Excli J. 15: 482-499.

Schmitz, H., Fromm, M., Bentzel, C.J., Scholz, P., Detjen, K., Mankertz, J., Bode, H., Epple, H.J., Riecken, E.O., and Schulzke, J.D. (1999). Tumor necrosis factor-alpha (TNFalpha) regulates the epithelial barrier in the human intestinal cell line HT-29/B6. J. Cell Sci. 112: 137-146.

Sen, R., and Baltimore, D. (1986). Multiple nuclear factors interact with the immunoglobulin enhancer sequences. Cell 46(5): 705-716.

Sepehri, S., Kotlowski, R., Bernstein, C.N., and Krause, D.O. (2007). Microbial diversity of inflamed and noninflamed gut biopsy tissues in inflammatory bowel disease. Inflamm. Bowel Dis. 13(6): 675-683.

Serra, D., Almeida, L.M., and Dinis, T.C.P. (2016). Anti-inflammatory protection afforded by cyanidin-3-glucoside and resveratrol in human intestinal cells via Nrf2 and PPAR-y: Comparison with 5-aminosalicylic acid. Chem Biol. Interact. 260: 102-109.

Shen, L., Black, E.D., Witkowski, E.D., Lencer, W.I., Guerriero, V., Schneeberger, E.E., and Turner, J.R. (2006). Myosin light chain phosphorylation regulates barrier function by remodeling tight junction structure. J. Cell Sci. 119(10): 2095-2106.

Shen, Z.-H., Zhu, C.-X., Quan, Y.-S., Yang, Z.-Y., Wu, S., Luo, W.-W., Tan, B., and Wang, X.-Y. (2018). Relationship between intestinal microbiota and ulcerative colitis: mechanisms and clinical application of probiotics and fecal microbiota transplantation. World J. Gastroenterol. 24(1): 5-14.

Shin, D.Y., Lu, J.N., Kim, G.Y., Jung, J.M., Kang, H.S., Lee, W.S., and Choi, Y.H. (2011). Anti-invasive activities of anthocyanins through modulation of tight junctions and suppression of matrix metalloproteinase activities in HCT-116 human colon carcinoma cells. Oncol. Rep. 25(2): 567-572.

Stallmach, A., Giese, T., Schmidt, C., Ludwig, B., Mueller-Molaian, I., and Meuer, S.C. (2004). Cytokine/chemokine transcript profiles reflect mucosal inflammation in Crohn's disease. Int. J. Colorectal Dis. 19(4): 308-315.

Tang, Y., Clayburgh, D.R., Mittal, N., Goretsky, T., Dirisina, R., Zhang, Z., and et al (2010). Epithelial NF-kappaB enhances transmucosal fluid movement by altering tight junction protein composition after $\mathrm{T}$ cell activation. Am. J. Pathol. 176(1): 158-167.
Taverniti, V., Fracassetti, D., Bo, C.D.e.I., Lanti, C., Minuzzo, M., KlimisZacas, D., Riso, P., and Guglielmetti, S. (2014). Immunomodulatory effect of a wild blueberry anthocyanin-rich extract in human Caco2 intestinal cells. J. Agric. Food Chem. 62(33): 8346-8351.

Tian, T., Wang, Z., and Zhang, J. (2017). Pathomechanisms of oxidative stress in inflammatory bowel disease and potential antioxidant therapies. Oxid. Med. Cell. Longev. 2017: 18.

Turner, M.D., Nedjai, B., Hurst, T., and Pennington, D.J. (2014). Cytokines and chemokines: at the crossroads of cell signalling and inflammatory disease. Biochim. Biophys. Acta - Mol. Cell Res. 1843(11): 25632582.

Ulluwishewa, D., Anderson, R.C., Mcnabb, W.C., Moughan, P.J., Wells, J.M., and Roy, N.C. (2011). Regulation of tight junction permeability by intestinal bacteria and dietary components. J. Nutr. 141: 769-776.

van Duynhoven, J., Vaughan, E.E., Jacobs, D.M., Kemperman, R.a., van Velzen, E.J.J., Gross, G., and et al (2011). Metabolic fate of polyphenols in the human superorganism. Proc. Natl. Acad. Sci. USA 108(Suppl 1): 4531-4538.

Van Itallie, C.M., Fanning, A.S., Holmes, J., and Anderson, J.M. (2010). Occludin is required for cytokine-induced regulation of tight junction barriers. J. Cell Sci. 123(16): 2844-2852.

Velikova, T., Kyurkchiev, D., Spassova, Z., Karakolev, I., Ivanova-Todorova, E., Altankova, I., and Stanilova, S. (2017). Alterations in cytokine gene expression profile in colon mucosa of inflammatory bowel disease patients on different therapeutic regimens. Cytokine 92: 12-19.

Vendrame, S., Guglielmetti, S., Riso, P., Arioli, S., Klimis-Zacas, D., and Porrini, M. (2011). Six-week consumption of a wild blueberry powder drink increases bifidobacteria in the human gut. J. Agric. Food Chem. 59(24): 12815-12820.

Vitaglione, P., Donnarumma, G., Napolitano, A., Galvano, F., Gallo, A., Scalfi, L., and Fogliano, V. (2007). Protocatechuic acid is the major human metabolite of cyanidin-glucosides. J. Nutr. 137: 2043-2048.

Vounotrypidis, P., Kouklakis, G., Anagnostopoulos, K., Zezos, P., Polychronidis, A., Maltezos, E., Efremidou, E., Pitiakoudis, M., and Lyratzopoulos, N. (2013). Interleukin-1 associations in inflammatory bowel disease and the enteropathic seronegative spondylarthritis. Auto Immun. Highlights 4(3): 87-94.

Waetzig, G.H., Seegert, D., Rosenstiel, P., Nikolaus, S., and Schreiber, S. (2002). p38 mitogen-activated protein kinase is activated and linked to TNF- $\alpha$ signaling in inflammatory bowel disease. J. Immunol. 168(10): 5342-5351.

Wang, L., Walia, B., Evans, J., and Andrew, T. (2010). IL-6 induces NF-kappa B activation in the intestinal epithelia. J. Immunol. 171(6): 31943201

Wang, R., Li, S., and Ho, C.-T. (2018). Absorption of polymethoxyflavones and their derivatives. J. Food Bioactives 2: 82-90.

Weber, C.R., Raleigh, D.R., Su, L., Shen, L., Sullivan, E.A., Wang, Y., and Turner, J.R. (2010). Epithelial myosin light chain kinase activation induces mucosal interleukin-13 expression to alter tight junction ion selectivity. J. Biol. Chem. 285(16): 12037-12046.

Wiczkowski, W., Romaszko, E., and Piskula, M.K. (2010). Bioavailability of cyanidin glycosides from natural chokeberry (Aronia melanocarpa) juice with dietary-relevant dose of anthocyanins in humans. J. Agric. Food Chem. 58(23): 12130-12136.

Willson, K., and Situ, C. (2017). Systematic review on effects of diet on gut microbiota in relation to metabolic syndromes. J. Clin. Nutr. Metab. 1(2): 1-12.

Woodward, G., Kroon, P., Cassidy, A., and Kay, C. (2009). Anthocyanin stability and recovery: Implications for the analysis of clinical and experimental samples. J. Agric. Food Chem. 57(12): 5271-5278.

Wu, T., Grootaert, C., Voorspoels, S., Jacobs, G., Pitart, J., Kamiloglu, S., and et al (2017). Aronia (Aronia melanocarpa) phenolics bioavailability in a combined in vitro digestion/Caco-2 cell model is structure and colon region dependent. J. Funct. Foods 38: 128-139.

Wu, J.-C., Tung, Y.-C., Zheng, Y-N., Tsai, M.-L., Ho, C.-T., and Pan, M.-H. (2018). 5-Demethylnobiletin is more effective than nobiletin in preventing AOM/DSS-induced colorectal carcinogenesis in ICR mice. J. Food Bioactives 2: 98-103.

Xiao, X., Kim, J., Sun, Q., Kim, D., Park, C.S., Lu, T.S., and Park, Y. (2015). Preventive effects of cranberry products on experimental colitis induced by dextran sulphate sodium in mice. Food Chem. 167: 438-446. 
Xie, L., Lee, S.G., Vance, T.M., Wang, Y., Kim, B., Lee, J.Y., Chun, O.K., and Bolling, B.W. (2016). Bioavailability of anthocyanins and colonic polyphenol metabolites following consumption of aronia berry extract. Food Chem. 211: 860-868.

Yi, W., Akoh, C.C., Fischer, J., and Krewer, G. (2006). Absorption of anthocyanins from blueberry extracts by Caco-2 human intestinal cell monolayers. J. Agric. Food Chem. 54(15): 5651-5658.
Yu, H., Wu, L.H., Xu, Z.L., Dong, D., and He, S.A. (2011). Protective effect of anthocyanins extract from blueberry on TNBS-induced IBD model of mice. Evidence-Based Complement. Altern. Med. 2011: 525462.

Zolotarevsky, Y., Hecht, G., Koutsouris, A., Gonzalez, D.E., Quan, C., Tom, J., Mrsny, R.J., and Turner, J.R. (2002). A membrane-permeant peptide that inhibits MLC kinase restores barrier function in in vitro models of intestinal disease. Gastroenterology 123(1): 163-172. 